gender, ethnicity, deprivation, BMI category, smoking status, the number of long-term conditions, the prevalence of 16 conditions, housing tenure, benefits received and housing occupancy.

Results For the adult residents of B\&D between 1st April 2016 and 31st March 2017, health and care costs were $£ 2,662$ (CI $£ 1,595, £ 3,729$ ) higher for people registered in their primary care records to have a carer $(n=1,295)$ compared to a matched cohort of adults who were not registered as having a carer. Social care accounted for the majority of this difference (39\%).

Conclusion The increased costs across all settings of care suggest that informal carers do not provide care substitution or, at least, that additional service use induced by the carer may dominate any substitution effect. There is an opportunity to provide bespoke training and education to informal carers and identify interventions that could support more care substitution.

For people who have a carer, such support may be a key element in enabling them to access services and, as such, there may be wider inequalities in access to services for people without a carer. In a society that is ageing with projections suggesting that there will be more people without carers in the future, these inequalities need to be addressed.

\section{P93 AGEING WELL IN RURAL COMMUNITIES: THE CONTRIBUTION OF NEIGHBOURHOOD PLANNING IN ENGLAND}

${ }^{1} S$ Buckner*, ${ }^{2} \mathrm{~A}$ Barnes, ${ }^{2} \mathrm{H}$ Jordan, ${ }^{1} \mathrm{C}$ Lee, ${ }^{1} \mathrm{C}$ Mattocks, ${ }^{3} \mathrm{E}$ Oliver, ${ }^{4} \mathrm{D}$ Pope, ${ }^{1} \mathrm{~L}$ Lafortune. ${ }^{1}$ Cambridge Institute of Public Health, University of Cambridge, Cambridge, UK; ${ }^{2}$ School of Health and Related Research, University of Sheffield, Sheffield, UK; ${ }^{3}$ School of Applied Social Sciences, Durham University, Durham, UK; ${ }^{4}$ Department of Public Health and Policy, University of Liverpool, Liverpool, UK

\subsection{6/jech-2020-SSMabstracts. 185}

Background Recent years have seen a proliferation of efforts globally to make cities places that foster healthy ageing. Despite exceptions, initiatives to promote the age-friendliness of rural communities have lagged behind. Identifying mechanisms that can enhance ageing well in rural settings and contribute to reducing inequalities is vital, particularly as these communities are experiencing rapid population ageing. This paper presents findings from a study that explored the potential of Neighbourhood Planning (NP) in England to generate processes and deliver outcomes in rural communities that support ageing well. NP is a participatory planning process that involves communities taking an active role in shaping their area.

Methods A scoping review was carried out to identify the existing evidence on NP and ageing and health in rural areas. Rural communities at an advanced stage of NP were mapped using QGIS to assess national coverage, including in relation to area deprivation and population age profile. All contactable communities were sent a questionnaire designed to identify those where NP focused on ageing-related issues. Six communities with an age-friendly focus in NP were selected as case studies where interviews and focus groups with stakeholders and residents and documentary analysis were carried out.

Results No literature that combined a focus on NP and ageing and rural settings was found. There were 572 rural communities at an advanced stage of NP. Mapping highlighted gaps in their distribution across rural England that broadly coincided with local authority districts with older age profiles and greater deprivation. The survey response was $n=75 / 557$. Respondents mentioned as ageing-related priorities in NP meeting older residents' housing needs $(n=30)$, supporting older residents' health and wellbeing $(n=23)$, and improving transport $(n=17)$. The survey informed the case studies. Findings from the latter indicated the potential of NP to result in age-friendly improvements in the physical environment, e.g. age-appropriate housing and improved walkways. They also highlighted various ways in which the very process of NP can support ageing well, e.g. by generating involvement of older people that can strengthen social connectedness. A detailed Theory of Change was developed of ways in which NP can support ageing well in rural communities.

Conclusion This study examined an under-researched mechanism for turning rural settings into places where people can age well. As well as highlighting the potential of NP to support the creation of age-friendly rural communities, it has delivered an evidence-informed Theory of Change that can be tested further.

\section{\begin{tabular}{|l|l}
\hline P94 THE FEASIBILITY AND ACCEPTABILITY OF DIGITAL \\
\hline
\end{tabular} TECHNOLOGY FOR HEALTH AND WELLBEING IN SOCIAL HOUSING COMMUNITIES IN CORNWALL: A QUALITATIVE SCOPING STUDY}

SA Buckingham, TW Walker*, K Morrissey. European Centre for Environment and Human Health, University of Exeter, Truro, UK

\subsection{6/jech-2020-SSMabstracts. 186}

Background Digital technology interventions for health and wellbeing are appealing, accessible, scalable and low cost. However, the evidence is far from established; many existing studies are of low quality, and there has been a focus on quantitative evaluation and on clinical and residential care populations. The Smartline project aims to explore the role that digital technology may play in improving health, wellbeing and social connectedness in social housing residents from low socioeconomic status communities in Cornwall. The aims of this study were: to explore existing digital technology use and competence; to explore digital willingness and readiness to use new technologies; to identify perceived barriers and facilitators to technology use; and to scope the feasibility and acceptability of potential digital interventions in the Smartline cohort.

Methods Three semi-structured focus groups (19 participants) and individual telephone interviews ( $\mathrm{n}=3$, ongoing) in a purposive, maximal variation sample of Smartline participants. To complement the qualitative responses, some self-reported quantitative data was collected in the focus groups, including perceived technology competence (selfrated according to the UK government Digital Inclusion Scale) and preference ranking of eight interventions. Focus groups and interviews were audio-recorded, transcribed verbatim and analysed thematically.

Results Existing levels of experience with digital technology are diverse, ranging from 'willing and unable' to 'expert'. Perceived barriers include usability, cost, poor internet connection, lack of skills and knowledge, safety and privacy concerns, sensory impairments and physical disabilities. Facilitators include awareness, training and technical support, improved access to 
facilities, and availability of choice. Preferred types of digital technology were wearable activity monitors (e.g. Fitbit ${ }^{\boxplus}$ ), virtual assistants (e.g. Amazon Alexa) and social messaging (e.g. WhatsApp). Analysis is ongoing, but overarching themes include: a need to improve awareness (what technologies are available and their capabilities for improving health and wellbeing); a willingness to learn (new skills and/or improve existing skills); the need for human contact (this should not be replaced by technology); and individual preferences (the importance of tailoring and choice).

Conclusion There is a need and desire to use digital technology to improve heath, wellbeing and social connectedness in the diverse group of Smartline participants. Barriers may be overcome with increased awareness and training, and tailoring and choice of interventions to meet individual needs and preferences. The findings will be used to inform piloting of different digital interventions in the next phase of the Smartline study. 